\title{
Study on Routing Protocol Used for Underground Coal Mine WSN based on Energy Cluster Head Election
}

\author{
Lei Zhang $^{1, a}$, Xin Wang ${ }^{1, b}$, Shu-guang Miao ${ }^{1, c}$, Duan Zhao ${ }^{2, c}$, Chun-xu \\ Chen ${ }^{1, c}$ \\ ${ }^{1}$ College of Information and Electrical Engineering, China University of Mining and Technology, \\ Xuzhou 221008, China \\ ${ }^{2}$ IOT Perception Mine Research Center, China University of Mining and Technology, Xuzhou \\ 221008, China; \\ az76557720@163.com,,Win_xin@cumt.edu.cn, ${ }^{\mathrm{c}}$ Clee_iot@cumt.end.cn
}

Key words: Wireless Sensor Networks; Routing protocol Load balancing; Cluster head selection Abstract. For underground wireless sensor networks with limited energy problem, this paper proposes a method based on AP cluster head routing protocol (EDAP). By computing energy consumption between both node to node and note to sink node, get the competition degrees $W$ that is a cluster basis. Use the $W$ to select the most suitable cluster head for data transmission from AP clustering algorithm. The simulation results show that it has a good stability EDAP and clustering uniform, which can significantly improve the survival time of the network sensor networks.

\section{Introduction}

In recent years, with the popularization and application of wireless sensor network in the coal mine, many fields for example the personnel position in coal mine have been developed vigorously. However, the harsh environment and the high cost of maintenance, especially the limited node energy are increasingly becoming a bottleneck for its further development. Therefore, how to reduce overall network energy consumption and prolong the network lifetime, have attracted the attention of experts and scholars ${ }^{[1]}$.

When it is practical application in the coal mine, the narrow tunnel and long distance from sink node make it difficult for normal node to communicate with sink node without the help of amplification, relay and other means. It brings high communication energy consumption and short network lifetime. In order to reduce communication energy and load balancing, you must be a large number of cluster nodes, each cluster select a cluster head, all of the information collected by the cluster head in the cluster to the Sink node [2].

Many researchers put forward a variety of cluster head selection method. The Leach protocol is a typical method. The protocol adopts the cluster head rotation mechanism, which reduces the energy consumption of communication and prolongs the network lifetime effectively. However, the Leach protocol also has disadvantages. For example, cluster heads are selected randomly so that a small residual energy of nodes are selected as the cluster heads. What's more, the cluster head node will consume more energy than other nodes. If a node with little energy is selected as the cluster head, the node will die early. This cannot achieve good network load balancing, not along extending the lifetime of WSN.

In order to improve the Leach, UCDP protocol is proposed in the literature [6]. Before transferring data in every round, UCDP protocol will divide dynamic the area into different parts, which can effectively balance the network load. However, this algorithm only considers the energy consumption between the nodes in its cluster. The energy consumption between the cluster head 
node and sink node isn't considered. At the same time, the core of this algorithm is Leach, which means a low rate of convergence.

When it comes to choosing a cluster head, both the energy consumption of a node and the rest energy of a node should be considered. At the same time, the rate of convergence can't be ignored. Therefore, based on AP algorithm and WSN load balancing, this paper proposes a cluster head selection protocol for WSN. And we call this protocol as EDAP. The EDAP will compare the energy consumption of node-to-node and the energy consumption of node-to-sink node before choosing a new cluster head. This realizes adaptive clustering. The core of EDAP is the AP algorithm, so the convergence rate is quick. Compared to Leach and UCDP, the results show that EDAP can prolong the network life cycle significantly.

\section{Network and the energy consumption model}

\section{network topology}

The routing protocol of WSN is related to the network topology. It can be divided into flat routing protocol and clustering routing protocol. Clustering network model is shown in figure 1 . Cluster head nodes gather data from normal node and then transfer the integration data to the sink node.

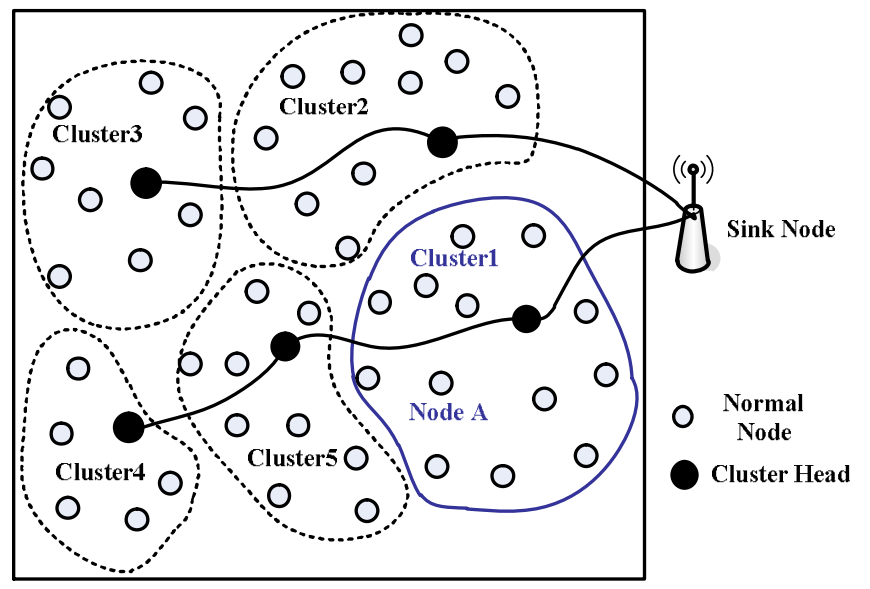

Fig.1 Clustering network model

In this paper, all the nodes in WSN are assumed as following ${ }^{\text {[8]: }}$

(1)The energy of all the nodes is heterogeneous, and the total energy of the wireless sensor network (WSN) is $E_{\text {all }}$. At the same time, the node can obtain its own energy $E_{s}$

(2)Each node is an independent node, which means any node can be a normal node as well as a cluster head node.

(3)At $t$ moment, all of the cluster head nodes make up a s set. At $d$ moment after the $t$ moment, all the nodes in s set can't be selected as a new cluster head.

(4)Every node works independently. When a node is receiving data or sending data, it doesn't affect the other nodes.

(5)Sending or receiving one bit information consumes $E_{\text {elec }}$ from the sending and receiving circuit

(6)Sending one bit data to per unit area consume $\varepsilon_{\text {amo }}$ of the emission amplifier.

(7)Every bit data fusion in cluster head node will consume $E^{D A}$.

(8) the sink node has a strong ability of calculation and its energy is infinite.

\section{energy consumption model}

This paper uses a simple energy consumption model. In this model, only the energy consumption of sending data and the energy consumption of receiving data are taken into consideration. Sending 
energy consumption includes $E_{T x-a n p}(1, d)$ ( The power consumption of the amplifier circuit) and $E_{T_{X}-\text { elec }}(1)$ (the energy consumption of sending circuit).

\section{(1) The energy consumption of sending data}

When a node send 1 bit data to another node and the distance between this two nodes is d, the energy consumption of this node is:

$$
\begin{aligned}
E_{T x}(l, d)= & E_{T x-\text { amp }}(l, d)+E_{T x-\text { elec }}(l) \\
& \begin{cases}E_{T x}(l, d)=E_{T x-\text { amp }}(l, d)+E_{T x-\text { elec }}(l) & d<d_{0} \\
E_{T x}(l, d)=l^{*} E_{\text {elec }}+l^{*} \varepsilon_{f s} d^{2} & d>d_{0}\end{cases}
\end{aligned}
$$

Where $d_{0}$ is distance threshold value, $E_{\text {elec }}, \varepsilon_{f s}, \varepsilon_{\text {amp }}$ are constants, $l$ is data length, which is depend on the actual performance of the node.

\section{(2) The energy consumption of receiving data}

Only the receiving circuit is working when the node is receiving data. Therefore, the energy consumption of receiving 1 bit data is:

$$
E_{R x}=E_{R x-\text { elec }}(l)=l * E_{\text {elec }}
$$

\section{(3)Gathered data energy consumption of the cluster}

The cluster head intergated the data from the normal nodes. The energy consumption of every bit is:

$$
E_{\text {aggregation }}(n, 1)=n 1 E_{D A}
$$

Where $E_{D A}$ is the energy consumption of gathering 1 bit data.

\section{(4) Energy consumption of the sensor}

The ADC, MCU and sensor part in the node gathering 1 bit data consume energy as:

$$
E_{\text {sense }}(1)=1 * E_{\text {sense }}
$$

\section{AP algorithm and EDAP}

\section{AP algorithm}

AP algorithm is one of the clustering algorithms ${ }^{[9-11]}$. Based on the fact that data is transferred between adjacent nodes, this algorithm will find a set of clustering center node. The clustering center nodes are also called exemplars. So, the best clustering effect can be achieved, which means the largest similarity of every node to its clustering center.

The credibility $\mathrm{r}(\mathrm{i}, \mathrm{k})$ and availability a $(\mathrm{i}, \mathrm{k})$ are introduced in the AP algorithm. The k node is the clustering center node of $\mathrm{i}$ node. The $\mathrm{r}(i, k)$ means how suitable the $\mathrm{k}$ node is a clustering center for the I node. The a $(i, k)$ means how well the I node chooses the $\mathrm{k}$ node as its clustering center. For all the nodes, if there is a node that the credibility and the availability is the biggest, the node will be the clustering center. $\arg \max (a(i, k)+r(i, k))$. The cluster progress of AP algorithm is updating the credibility and availability constantly. The progress is following:

$$
\begin{array}{r}
r(i, k) \leftarrow s(i, k)-\max _{k^{\prime} \neq k}\left\{a\left(i, k^{\prime}\right)+s\left(i, k^{\prime}\right)\right\} \\
\text { If } i \neq k \quad a(i, k) \leftarrow \min \left\{0, r(k, k)+\sum_{i \notin\{i, k\}} \max \left\{0, r\left(i^{\prime}, k\right)\right\}\right\} \\
a(k, k) \leftarrow \sum_{i \neq k} \max \left(0, r\left(i^{\prime}, k\right)\right)
\end{array}
$$


To void shock, we introduce the damping coefficient $\lambda \in[0,1]$ in this algorithm. We can adjust the damping coefficient to eliminate the shock. In the process of each iteration, the current iteration results and the previous iteration results are weighted summed. When the current number of iteration is $t$, the weighted formula is:

$$
\begin{aligned}
& r^{(t)}(i, k)=(1-\lambda) \times\left(s(i, k)-\max _{k^{\prime} \neq k}\left\{a^{(t-1)}(i, k)+s\left(i, k^{\prime}\right)\right\}\right)+\lambda \times r^{(t-1)}(i, k) \\
& \text { If } i \neq k, \\
& a^{(t)}(i, k)=(1-\lambda) \times\left(\min \left\{0, r^{(t-1)}(k, k)+\sum_{i \neq\{i, k\}} \max \left\{0, r^{(t)}\left(i^{\prime}, k\right)\right\}\right\}\right)+\lambda \times a^{(t-1)}(i, k) \\
& a^{(t)}(k, k)=(1-\lambda) \times\left(\sum_{i \neq\{i, k\}} \max \left\{0, r^{(t)}\left(i^{\prime}, k\right)\right\}\right)+\lambda \times a^{(t-1)}(i, k)
\end{aligned}
$$

\section{Introduction of EDAP algorithm}

In the cluster progress of AP algorithm, a similarity matrix is input. The value in the similarity matrix represents the similarity between two nodes ${ }^{[13-15]}$. When choosing a cluster by using the EDAP algorithm, both the energy consumption and the current energy should be considered. The cluster node should meet the following conditions:

1) It consumes less energy when the others nodes send data to the cluster head node.

2) Its residual energy is more than others.

3) It consumes less energy when this cluster send data to the sink node.

In this paper, the Weight ( $W$, Competitiveness) is the input of the EDAP algorithm. the $\mathrm{W}$ can reflect the competitive ability of each node. The formula ${ }^{[10]}$ is:

$$
w_{i j}=-l\left(E_{\text {elec }}+\varepsilon_{f s} d_{i j}{ }^{2}+\varepsilon_{f s} d_{j}\right) * \exp \left(\frac{1}{W_{j}}\right), \quad w_{j}=\frac{E_{\text {current } j}}{E_{\text {current }}}
$$

Where, $\left(E_{\text {elec }}+\varepsilon_{f s} d_{i j}{ }^{2}+\varepsilon_{f s} d_{j}\right)$ contains two parts energy consumption. One is the communication consumption between the normal node and the cluster node. The other is the communication consumption between the cluster node and the sink node. Obviously, the less energy consumption in this part, the better performance this algorithm has. when the sending circuit sends 1bit data, the energy consumption is $\mathrm{E}_{\mathrm{elec}} . \boldsymbol{\varepsilon}_{f_{S}} d_{i j}{ }^{2}$ is the energy consumption of emission amplifier. It is better for extending the life of the WSN if less energy is consumed in this part. In the formula(12), the $\exp \left(1 / W_{j}\right)$ can increase the difference of $W . W_{j}$ is the ratio of the residual energy of $\mathbf{j}$ node and the average residual energy of all the nodes. A bigger $W_{j}$ means more residual energy of $j$ node. Smaller $\left(E_{\text {elec }}+\varepsilon_{f s} d_{i j}{ }^{2}+\varepsilon_{f s} d_{j}\right)$ and $\exp \left(1 / W_{j}\right)$ illustrate that the $w_{j}$ is bigger, which means the more residual energy of $\mathrm{j}$ node. Based on that, the $\mathrm{j}$ node is suitable for being a cluster node. That will reduce the death of node and the life of the network will be longer.

\section{Principle of EDAP}

The specific principle of EDAP is following:

(1) Wij can be derived from the formula (12). Wij can represent the similarity between different nodes in the similar matrix.

(2) the initial values of $r(i, j)$ and $a(i, j)$ are 0 . The maximum number of iterations is set. The cluster center is selected after a certain number of iterations.

$$
\begin{aligned}
& r(i, j)=W_{i j}-\max _{j . s . t . j \neq j}\left\{a\left(i, j^{\prime}\right)+W_{i j}\right\} \\
& a(i, j)=\min \left\{0, r(j, j)+\sum_{i \text {..s.t. } i \notin\{i, j\}} \max \left\{0, r\left(i^{\prime}, j\right)\right\}\right\}
\end{aligned}
$$

(3) put the sum of $r(i, j)$ and $a(i, j)$ into a new matrix. The maximum on the diagonal of the 
matrix corresponds to the cluster head nodes.

\section{EDAP flow}

(1) Calculate the distance between any two nodes and put the distance into a $\mathrm{N}^{*} \mathrm{~N}$ matrix $\mathrm{D}$. Then Wij can be derived from the formula (12) according to the matrix D.

For any $j$, the value of $W_{i j}$ is $E_{0}$ at the first round of choosing cluster node. The $W_{i j}$ will be put into a $\mathrm{N}^{*} \mathrm{~N}$ matrix $\mathrm{W}$.

(2) The initial values of $r(i, j)$ and $a(i, j)$ are set as 0 . The $r(i, j)$ and $a(i, j)$ are put into matrix $\mathrm{A}$ and matrix $\mathrm{R}$ respectively. The matrix $\mathrm{E}$ is the sum of matrix $\mathrm{A}$ and matrix $\mathrm{B}$. The biggest iterations is set. $r(i, j)$ and $a(i, j)$ are iterated and updated by formula (13) and (14) until a set number of iterations reaches the maximum number of iterations

(3) Find the j point which meets $E(j, j)>0$, and select the point as the cluster head.

(4) Calculate the energy consumption of cluster node. The energy consumption of cluster head node includes three parts: gathering the information of normal nodes, integrating received data and sending the integrated information to the sink node. At the same time, the energy consumption of normal nodes sending information to cluster node is calculated.

(5) The energy of each node reduces after every round. $E_{\text {currentj }}$ is the energy that the initial energy at every round minus the consumption energy at this round. If there are $\mathrm{k}$ death nodes, the number of death nodes will plus $\mathrm{K}$ and enter the next round.

(6) Recalculate all the $W_{i j}$ according the residual energy of each node that getting from the precious round. Then the matrix $\mathrm{W}$ is updated. The algorithm will go back to step(2) and continue to execute the following step until all the nodes die.

EDAP keeps the balance of energy consumption of every nodes. This algorithm can select the most suitable node as the cluster head node by the competitiveness. This increases the utilization efficiency of every node. Therefore, EDAP algorithm can keep the balance of network load and make more efficient use of energy

\section{Simulation analysis}

\section{Simulation parameter}

We use the MATLAB to simulate. The range of simulation is $200 * 200 \mathrm{~m}$. The initial parameters is shown in table 1 .

Table. 1 simulation parameters

\begin{tabular}{cc}
\hline parameters & value \\
\hline Sink & $(0,0)$ \\
$E_{\text {all }}$ & $200 \mathrm{~J}$ \\
Packet length & $4000 \mathrm{bit}$ \\
ctrPacketLength & $100 \mathrm{bit}$ \\
$E_{\text {elec }}$ & $5 \mathrm{~nJ} / \mathrm{bit}$ \\
$E^{D A}$ & $5 \mathrm{~nJ} / \mathrm{bit}$ \\
$\varepsilon_{\text {amo }}$ & $100 \mathrm{Pj} / \mathrm{bit} / \mathrm{m}^{2}$ \\
\hline
\end{tabular}

\section{Results compared}

With the continuous cycle, all the node could die randomly. Based on the same set of random data, EDAP and Leach are compared under the situation that all nodes has survived. Comparing the two pictures in the fig.2, we can see that EDAP has a better performance in uniformity of clustering than Leach. According to relevant researches, the more uniform the clustering is, the longer life the network will have. Therefore, EDAP can contribute to prolong the network lifetime. 


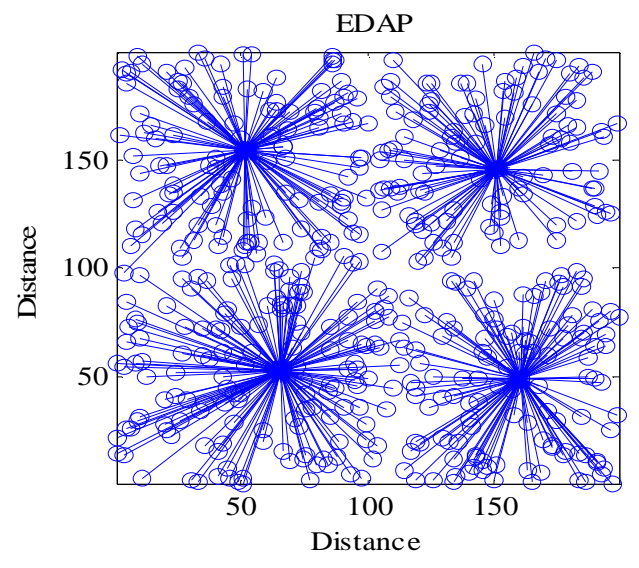

Fig.2(a) Clusters effect of UDAP

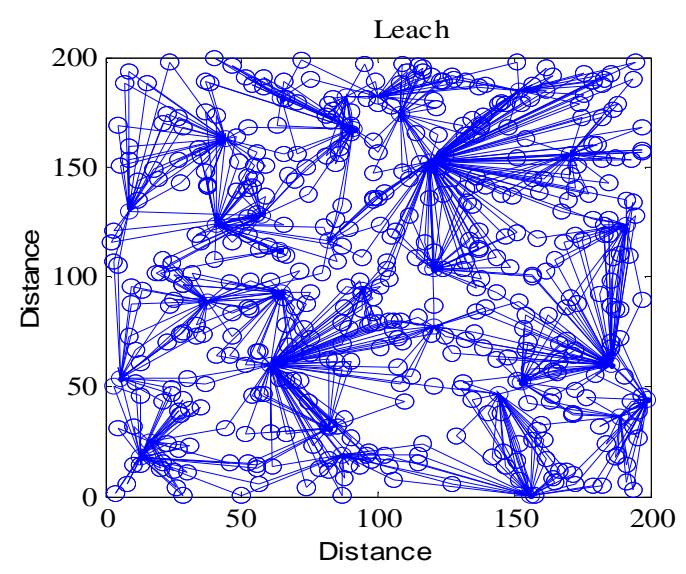

Fig.2 (b) Clusters effect of Leach

\section{The comparison of network survival time}

The fig. 3 shows the relationship between the number of surviving nodes and rounds. In the literature[16], the network doesn't work when the number of nodes in the network is less than $90 \%$ of the total number of nodes. Therefore, the simulation only consider the situation that the surviving nodes are more than $90 \%$ of the total number of nodes. As can be seen from fig.3, UDCP and EDAP are superior to Leach from the perspective of energy. At the same time, EDAP considers both the energy between nodes and the energy from normal node to sink node. The EDAP algorithm has a better performance in energy balance. So, EDAP is superior to the other two algorithms whether from the time of first death node or overall survival time of network.

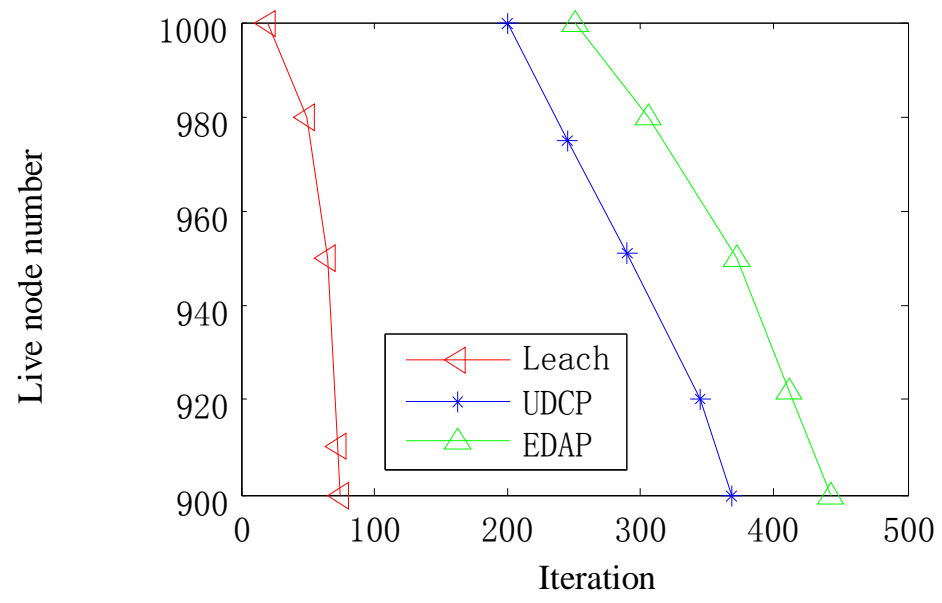

Fig.3 life cycle of the three protocols

\section{Comparison of total network energy and the node average remaining energy}

Fig. 4 shows the relationship between total network energy and rounds. The Leach curve is the steepest. The curves of UCDP and EDAP are relatively flat. The falling speed of EDAP is better than that of UDCP. EDAP fully considers both the energy consumption between nodes and the energy consumption between the node and the sink node. Based on that, EDAP is more effective to balance the energy consumption between nodes than the other protocols. Thereby, the network lifetime can be prolonged. 


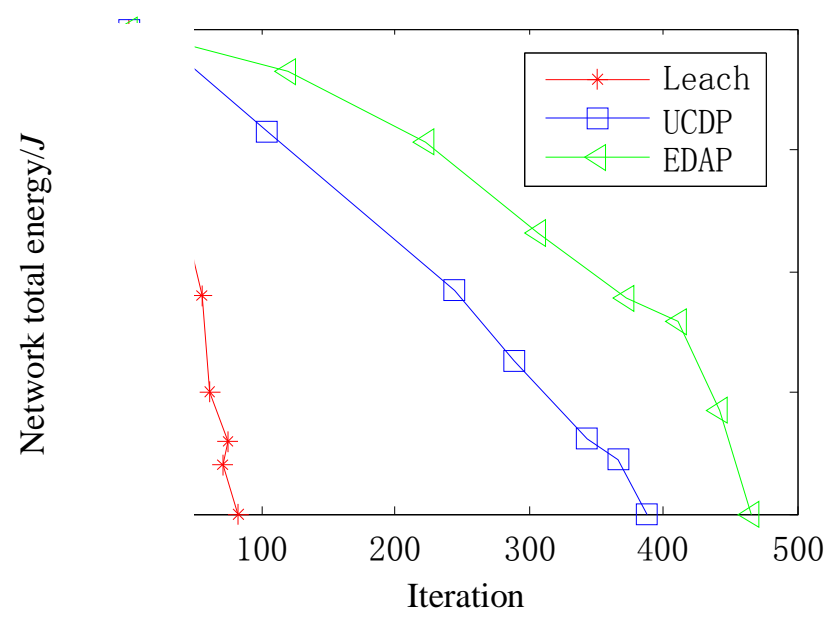

Fig. 4 the relationship between the total energy and the time

Fig. 5 shows the relationship between the average energy and round. The energy consumption of every round is average in the EDAP. However, the energy consumption of every round in the Leach declines precipitously. So EDAP can prolong the network lifetime.

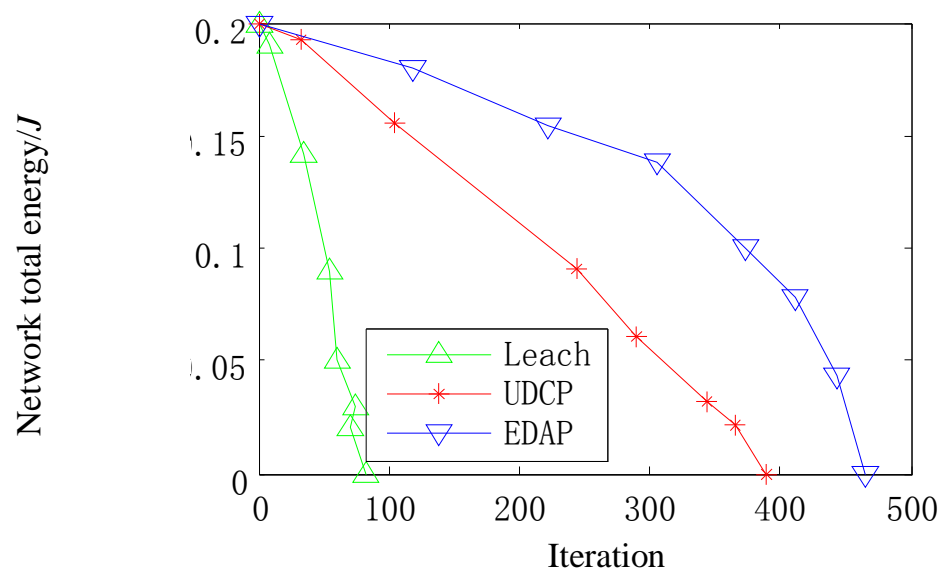

Fig.5 the relationship between the average energy and round

\section{Conclusion}

In the Leach algorithm, only the energy consumption between nodes is considered when selecting a cluster head node. In this paper, the EDAP algorithm is presented to improve LEACH algorithm. the EDAP algorithm considers not only the energy consumption between nodes but also the node position and the remaining energy. The simulation proves that EDAP can balance the network load and prolong the network lifetime. However, the energy consumption is so simple that it maybe not suitable for the coal mine.

\section{Acknowledgements}

This work was financially supported by the National Key Basic Research Program of China (973 Program, NO. 2014CB046300).

\section{References}

[1] Zhang X L, Liang Wei, Yu H B,etal. Survey of transmission scheduling methods in wireless sensor networks]J]. Journal on Communications,2012,33(5);143-157. 
[2] Saleh A M S, Borhanuddin Mohd A, Rasid M F A, et al. A self-optimizing scheme for energy balanced routing in Wireless Sensor Networks using SensorAnt.[J]. Sensors, 2012, 12(8):11307-11333.

[3] Echoukairi H, Bourgba K, Ouzzif M. A Survey on Flat Routing Protocols in Wireless Sensor Networks[M]// Advances in Ubiquitous Networking. Springer Singapore, 2016.

[4] A1-karaki J N,Kamal A E. Routing techniques in wireless sensor networks: A survey [J].IEEE Wireless Communication,2004,11(6):6-28.

[5] YAN Xin-fang, ZHANG Han, LI Ling, etal. EAMCT-G Optimization Algorithm Based on Lord Balance in WSN[J].Journal of Tianjin University, 2012,45(8): 735-739.

[6] SUN Yan-qing, PENG Jian, LIU Tang, CHEN Xiao-hai. Uneven clustering routing protocol based on dynamic partition for wireless sensor network[J].Journal on Communications, 2014,35(1):192-206.

[7] Heinzelman W, Chandrakasan A, Balakrishnan H. Energy Efficient Communication Protocol for Wireless Microsensor Networks [C] .Maui: IEEE Computer Society, Proceedings of the 33rd Hawaii International Conference on System Sciences, 2000. 3005 -3014

[8] LI B, WANG W J, YIN Q Y,etal.An energy-efficient geographic routing based on c-ooperative transmission in wireless sensor networks]J]. Sci China Inf Sci, 2013,56:757-4762.

[9] Alfons A, Croux C, Filzmoser P. Robust Maximum Association Estimators[J]. Journal of the American Statistical Association, 2016:1-29.

[10] Lee, Y. and S. Choi, Maximum within-cluster association[J]. Pattern recognition letters, 2005. 26(10):1412-1422.

[11] Steinley, D., K-means clustering: A half-century synthesis. British Journal of Mathe-matical and Statistical Psychology, 2006. 59(1): 1-34.

[12] XIAO yu , YU Jian. Semi-Supervised Clustering Based on Affinity Propagation Algo-rithm[J].Journal of Software,2008,19(11):2804-2806.

[13] Jain, A.K. Data clustering:50 years beyond K-means[J].Pattern recognition letters,2010.31(8):651-666.

[14] D. Izadi, J. Abawajy, and S. Ghanavati. Quality control of sensor network data[J].Advances in Automation and Robotics, 2012, 1(1): 467-480.

[15] Hongyan Cui, Chen Feng, Ren Ping Liu, etal Prolonging network lifetime in two-level heterogeneous Wireless Sensor Networks [A]. 2012 International Symposium on Communications and Information Technologies (ISCIT)[C], 2012, 871-876.

[16] QING L, ZHU Q X , WANG M W.A distributed energy-efficient clustering algorith $\mathrm{m}$ for heterogeneous wireless sensor networks[J].Journal of Software,2006,17(3):481-489 